\title{
Auge y caída de la hermenéutica: la crítica literaria en Estados Unidos luego del New Criticism
}

\author{
ELÍAS JOSÉ PALTI \\ Universidad de California en Berkeley-CONICET
}

Si comparamos con la situación reinante en Norteamérica en disciplinas humanísticas más tradicionales, como la historia y la filosofía (mcjores representantes de lo que allí se llama el mainstream académico), que continuaron siendo por largo tiempo bastiones del conservadurismo intelectual y refractarias a las novedades teóricas a las que supieron asimilar sólo en sus márgenes, el ámbito de la crítica literaria se caracteriza allí por ser escenario de tempranas intervenciones radicales. El tono iconoclasta que asume Susan Sontag (conocida como novelista y guionista de cine) en un artículo, cuyo título es ya elocuente, «Against Interpretation» (1964), resulta representativo del asalto global que se produce en los sixties contra el establishment académico representado por el $\mathrm{New}$ Criticism ${ }^{1}$. Dicho ataque resultará tan devastador (al punto de que aquella escuela ya no recobraría su anterior prestigio) como perturbador, puesto que llevaría a cuestionar la misma Iegitimidad de la empresa crítica, «En lugar de una hermenéutica necesitamos una erótica del arte", son las palabras con que Sontag cierra su artículo antes mencionado ${ }^{2}$. Éste era aún el clima general de ideas de los setenta, fuertemente polarizado entre corrientes antagónicas, cuando, por vía principalmente de la tradición hermenéutica, comienza la «invasión de la filosofía continental». E. D. Hirsch, Jr. ${ }^{3}$, será uno de los primeros en apelar al conjunto de herramientas conceptuales aportadas por la hermenéutica continental para mediar en esta contien- $\mathrm{da}^{4}$. Asi, a diferencia de lo que sucediera en Estados Unidos en otras disciplinas, como en la antropología, donde, de la mano de Geertz, la hermenéutica se asociaría a las corrientes teóricas más radicales, en el ámbito de la crítica literaria ésta aparecería como el espacio natural desde donde forjar un middle ground entre las corrientes antinómicas que se disputaban alli la primacía intelectual.

Hirsch partiría de premisas afines a las establecidas por el New Criticism, especialmente aquella fundamental para esa escuela que afirma la distinción radical entre texto y contexto, para terminar, sin embargo, enfatizando la importancia de la consíderación de la intencionalidad del autor en la comprensión de una determinada expresión literaria. Este autor aparece así como abogando por un modelo estrictamente filológico (por entonces, único modelo de respetabilidad académica) que, sin embargo, y aun cuando él mismo lo considera afin a los postulados del New Criticism (Hirsch dedica su Validity in Interpretation a Wimsatt y Crane), de hecho incorpora al mismo una dimensión pragmática (la consideración de la «intencionalidad» del autor) completamente ajcna a esta escuela. Pero, por otro lado, Hirsch intenta evitar las consecuencias relativistas a 'las que conduciría un contextualismo radical distinguiendo la eintencionalidad" del autor de su "contexto de emisión" (manteniéndose, así, dentro del plano puramente formal de los textos, como exigia el New Criticism). De este modo, Hirsch 
termina, en cierto modo, invirtiendo el sentido tradicional dado a aquellos conceptos por las filosofías del lenguaje.

En efecto, la intencionalidad del autor (normalmente considerada, en el ámbito de la lógica pragmática, como una función del contexto comunicativo en el que una expresión dada por el mismo tuvo lugar), Hirsch la reinterpreta (siguicndo una lec tura personal de las ideas de Frege) como el elemento invariable tras sus diversas posibles manifestaciones textuales. La intencionalidad del autor debe ser, para Hirsch, parafraseable de modo tal que pueda captarse su contenido semántico independientemente de la cxpresión particular en que ha sido emitida. «La sinonimia», dice, «depende de la determinación lentendida como la propiedad de ser algo determinado] del sentido, la emancipación del pensamiento de la prisión de una particular forma lingüistica» (The Aims of Interpretation, p. 10). La información contextual (el marco pragmático) importa, para Hirsch, a la significación (significance) de una expresión, no al sentido (meaning) de la misma. Sólo el primero es materia de interpretación, el scgundo, en cambio, hace al entendimiento. Mientras que el primero se orienta a la explicación de un sentido, el segundo busca simplemente «entender a éste en sus propios términos»; y si bien ambos constituyen objetos legitimos de estudio, en todos los casos el primero presupone necesariamente al segundo.

«Para comprender una expresión es, de hecho, no sólo posible sino absolutamente necesario entenderla en sus propios términos. Si no podemos distinguir un contenido de conciencia de su contexto, no podemos conocer ningún objeto en el mundon (Validity in Interpretation, p. 134).

Lo contrario conduce, para Hirsch, a la «falacia de la Cenicienta»: si el sentido cambiara permanentemente, «no habria forma de distinguir a la verdadera Cenicienta entre todas las contendientes... la nucva sandalia no calzaría más a la nueva Cenicienta» (Validity in Interpretation, p. 46).

Tal comprensión «en sus propios términos» no es, en realidad, más que un ideal, probablemente inalcanzable; pero, de todos modos, marca una direccionalidad para el progreso de nuestro conocimiento. Aun cuando nunca podamos desprendernos completamente de nuestros preconceptos, podemos sí verificarlos $y$ modificarlos en el caso de que una experiencia dada frustre nuestras expectativas previas. Hirsch interpreta esto como un proceso de aprendizaje (según la noción piagetiana de corrigibie schemata) por el cual escapamos al círculo de hierro de nuestras categorías actuales.

El cuestionamiento, que pronto se produce, al supuesto básico de la hermenéutica, es decir, de la posibilidad de la compresión de una determinada cultura cen sus propios términos», instalaría una auténtica fractura epistemológica en la disciplina (c inicia lo que allí se conoce como «el giro lingüístico"). En la obra de David Cousenz Hoy se pueden rastrear el tipo de aporías a las que la hermenéutica se ve enfrentada una vez que se problematiza el supuesto de la transparencia al propio autor del significado de su obra. En The Critical Circle ${ }^{5}$, Hoy contrapone a la versión hermenéutica «ingenua» de Hirsch (que él entiende de matriz ricoeuriana) la suya propia fundada en la idea hermenéutica «más sofisticada» de H.-G. Gadamer. Hoy denuncia en Hirsch lo que llama la "falacia intencionalista» (aunque de un modo distinto a como lo hiciera el $\mathrm{New}$ Criticism $)^{6}$, que reduce el texto a su proceso de producción, ignorando la serie de sus efectos y realizaciones efectivas (el «contexto de recepción») como una dimensión constitutiva del significado de un determinado texto. Con ello disloca la premisa básica de la hermenéutica de 
Hirsch, que se funda en la idea de la existencia de «sentidos culturales (individuales o colectivos) previos a toda interpretación (idea de la que deriva el concepto de autonomía semántica del referente).

Para Hoy, en primer lugar, kes el lenguaje el que hace posible algo como la intencionalidad, y no a la inversas (The Critical, p. 38). No hay acción que no sea significativa, y no hay significados articulables fuera de las estructuras del lenguaje. El lenguaje viene a ocupar entonces el lugar del autor como horizonte último y soporte a la vez de la inteligibilidad histórica; se convierte así en el fenómeno primitivo, autocontenido y constituyente ( $y$ no sólo constitutivo) de nuestra realidad social. Ahora bien, en segundo lugar, no hay tampoco para él «significados» fuera de, o anteriores a, sus interpretaciones, es decir, a las realizaciones verbales. $\mathrm{El}$ «lenguajes de la hermenéutica gadameriana ya no es, pues, el de la langue de Saussure, sino más bien el de su parole; la escurridiza historia de las comunicaciones efectivamente realizadas, y no el universo ya dado de las combinaciones significativas estructuralmente posibles. En fïn, la quiebra de la rígida distinción entre el texto y su contexto de recepción conduce a la disolución misma del objeto como tal, a la paradoja de un textualismo sin texto. Como señala Stanley Fish en su provocativo libro Is There a Text in this Class? ?, no habría "obras" sino únicamente la historia de sus lecturas por parte de comunidades interpretativas articuladas según sus propios estándares de evaluación antes que por las propiedades intrínsecas del texto o del acto intencional del autor cuya lectura aquéllos hacen posible ${ }^{8}$.

Esto no significa, sin embargo, para Hoy, que tales modos de leer sean subjetivos e idiosincráticos. Hoy sigue también aquí a Gadamer, quien explicara esto apelando al concepto aristotélico de phrónesis (sabiduría práctica), redefinido por él como la capacidad de «percibir lo que está en juego en una situación dadas (The Critical Circle, p. 58). Esta capacidad no se orienta al kconocimiento» (la *compren" sión en sus propios términos») sino al «entendimiento». El mismo presupone sienpre la aplicación (Anwendung) de un marco categorial dado (lo que Gadamer llama pre-juicios). Pero estos pre-juicios no nos encierran dogmáticamente en la celda de nuestros propios supuestos, éstos pueden ser, al menos, parcialmente clarificados en el proceso dialógico. La phrónesis exige que esta «aplicación» se vuelque sobre sí a fin de especificar el contexto comunicativo y posibilitar la comprensión mutua. De este modo puede emerger «un diálogo genuino [en el que] los pre-juicios pueden ser traídos a la conciencia y chequeados frente a sus ramificaciones en términos del asunto en cuestión mismo [la propia capacidad para comunicarse, para sostener un intercambio mutuamente inteligible]: si el pre-juicio se muestra inadecuado, entonces la unilateralidad en la interpretación que éste introduce [y que produce la falla comunicativa] puede ser expuesta, abriendo así el camino a nuevas interpretaciones" (7he Critical Circle, p. 77). Este mismo concepto se aplica cuando se trata de hechos o voces del pasado. El pasado, para Hoy, como para Gadamer, no es algo cerrado, mantiene su capacidad de sostener un diálogo con el presente en la medida en que ambos forman parte de una stradición" (proceso comunicativo sicmpre cn curso). El «entendimiento» supone la continuidad-discontinuidad de esta tradición. "Nosotros no vemos a Platón», ejemplifica Hoy, «como lo hacian Descartes y Kant, pero ciertamente vemos a Platón diferente a causa de Descartes y Kant) (The Critical Circle, p. 41). La historia existe como historia de los efectos (Wirkungen), de la serie de las modificaciones en la tradición que ella misma produce. $Y$ esta historia de efectos es la que a la vez hace posible (ya que articula dicha stradición» como tal) y necesaria (debido a que la misma cambia 
a través del tiempo) a la hermenéutica (si el pasado fuera completamente transparente, no necesitariamos una hermenéutica; si nos fuera completamente extraño, sería inviable).

La «solución» que ofrece Hoy parece, sin embargo, intentar sostenerse simultáneamente en dos proposiciones contradictorias entre sí. La primera, implícita en la idea de que podamos «chequear» nuestros pre-juicios, afirma lo que podemos llamar la «independencia semántica del referente», Como asegura Hoy en su respuesta a Fish, toda «teoría de la recepción» presupone un «texto" (en el sentido de algo situado más allá del horizonte de nuestras propias interpretaciones del mismo), de lo contrario, «no habría nada a lo que responder o al cual recepcionars (The Critical Circle, p. 158). Sin embargo, en su discusión con Hirsch, insiste en la idea de que «todo entendimiento presupone una interpretacions (The Critical Circle, p. 51). Sostener, por un lado, la absoluta independencia semántica del referente tornaría imposible el entendimiento. Como señala Hoy, para que exista comunicación, son necesarios «significados compartidos», es decir, significa afirmar que los participantes «comparten un lenguaje» (The Critical Circle, p. 62) ${ }^{9}$. Pero, por otro lado, ya no habría, en dicho caso, una verdadera "apertura" (idea que presupone la de la independencia semántica del referente, un stexto"), o, mejor dicho, una apertura parcial pero siempre dentro de la tradición compartida. Aquellos pre-juicios fundamentales que están en la base del proceso comunicativo (la tradición, en tanto que condición de posibilidad del mismo) no pueden ellos mismos convertirse en objeto de crítica. Toda comunicación sería, pues, siempre inevitablemente confirmatoria de la propia tradición (con lo que la postura de Hoy se haría pasible de las mismas críticas que recibiera su maestro, Gadamer, y a las que él trata aquí de superar, a saber, el adoptar una postura esencialmente conservadora) ${ }^{10}$.

Estas tensiones parecen insuperables para toda hermenéutica, puesto que el concepto dialógico, al mismo tiempo que necesita de un «referente» externo (de lo contrario, sería un monólogo), lo debe negar como tal (de lo contrario, no habría un entendimiento mutuo, verdadero diálogo) ". Hoy intenta, finalmente, escapar a este dilema introduciendo una distinción entre niveles. La vuelta hacia sî que se produce con la phrónesis envuelve un segundo nivel de creencias de una naturaleza completamente diferente de la de aquellas «creencias de primer nivel», inmediatamente involucradas en el intercambio comunicativo, dado que permiten su «puesta entre paréntesis». «La tesis historicista", asegura, «involucra creencias de segundo orden, o una "creencia acerca de creencias" (a veces llamada una "actitud", como distinta de la "creencia"). Como tal, confundirla con las creencias de primer orden para extraer de ello conclusiones nihilistas es una falaciay (The Critical Circle, p. 139).

Para Fish, lo que es falaz es esta misma distinción entre niveles de creencias. Tal ideal de «apertura» que Hoy propone es aún êl mismo una crecncia; y una creencia, además, ingenua. Toda «apertura» presupone ya un marco normativo, «uno no puede "desafiar las categorizaciones", sólo puede categorizar de un modo diferente» ${ }^{12}$. La decisión de kabrirse», ejemplifica Fish, lleva consigo la pregunta «iabrirse respecto a qué?" (uno no puede "abrirse" en general); "tal tipo de apertura», pues, «no es más (ni menos) que la resolución de ser diferentemente cerrado" (The New Historicism, p. 310). Así como la distinción entre «significación» y «sentido» de Hirsch no era más que un recurso que le permitía colocar al sujeto de una emisión por encima de su contexto de emergencia, la distinción en niveles que intenta Hoy no sería más que un intento análogo de colocar, 
esta vez, al sujeto-intérprete por encima de su propio contexto de emergencia (es decir, de las condiciones de recepción del sujeto-emisor de Hirsch). Esta «ingenuidad" tiene, sin embargo, para Fish sus raí" ces en el mismo contexto de emergencia al que se pretende negar, puesto que constituye una de las condiciones necesarias al funcionamiento de la institución profesional.

«Un profesional debe encontrar un modo de operar en el contexto de propuestas, motivaciones y posibilidades que lo preceden, e incluso lo constituyen, $y$ aun así mantener la convicción de que êl es "esencialmente el propietario de su propia persona y capacidades". El modo que él encuentra es el anti-profesionalismo... El profesionalismo es el emblema mismo de esta condición. El profesional que es "expresivo" en su propio pensamiento y acción de las instituciones, $y$, sin embargo, se "expresa" en el nombre de esencias que trascienden la institución y le proveen un punto de mira para la crítica, no está produciendo ninguna contradicción, sino simplemente actuando del único modo que pueden hacerlo los seres humanos ${ }^{13}$.

Para entender este afán de «apertura», es necesario, pues, internarse en los mecanismos por los que tales discursos críticos son intersubjetivamente producidos $y$ públicamente compartidos en el seno de comunidades interpretativas. Ello no conlleva, sin embargo, para Fish, una forma de subjetivismo. «Los significados y los textos producidos por una comunidad interpretativa», asegura Fish, «no son subjetivos porque no provienen de un individuo aislado sino de un punto de vista convencional y puiblicon (Is there?, p. 14). Dicho concepto «también explica», dice, "por qué hay desacuerdos y por qué ellos pueden ser debatidos de un modo regular: no debido a la estabilidad de los textos, sino a la estabilidad de la constitución de la comunidad interpretativas (Is there?, p. 15). Lo que para Fish definitivamente descarta tal explicación es la posibilidad de la traducibilidad de los sentidos correspondientes a comunidades lingüisticas diversas (lo que Hoy llamara, siguiendo a Gadamer, una «fusión de horizontes»), lo que no significa, sin embargo, para él, renunciar al derecho a pretender que su teoria (como pretende toda teoría) sca, al menos temporariamente, aceptada como verdadera.

al final, he renunciado tanto como reclamado la generalidad; la he renunciado porque renuncié a tratar de identificar el modo verdadero de lectura; la he reclamado porque sostengo mi derecho, como cualquier otro, de argumentar a favor de un modo de lectura, la cual, si es aceptada, podría, por un tiempo al menos, convertirse en la verdadera. En sintesis, he preservado la generalidad por medio de retorizarlas (Is there?, p. 16).

Sin embargo, aun la retórica presupone cierta inteligibilidad a fin de operar persuasivamente. Fish mismo señala esto cuando inmediatamente afirma que shabiendo redefinido la actividad crítica de modo que ésta no sea más un asunto de demostración sino de persuasión (infinitamente negociada), me enfrento a la tarea de dar cuenta, dentro del nuevo modelo, de todo aquello que ha sido constitutivo de la institución literaria: textos, autores, períodos, géneros, cánones, estándares, acuerdos, disputas, valores, cambios, etc.» (Is there?, p. 17). Esto implica que la «institución literaria» proveería un conjunto de catcgorias que servirian de sustrato común a las diferentes teorías. De lo contrario, si «todo aquello constitutivo de la institución literaria» se viera completamente redefinido dentro de cada comunidad interpretativa, no podría pensarse siquicra en intentar dar cuenta, dentro de la nueva teoría, de conceptos tales como «texto», «autor», etc, que fueron forjados por paradigmas extraños a la misma y sólo tienen sentido dentro de ella. Por otro lado, con tal concepto de "comunidades interpretativas» Fish mismo parece intro- 
ducir (esta vez, subrepticiamente) una dislinción de niveles similar a la propuesta por Hoy. Cabe al menos preguntarse si las mismas constituyen «referentes extra-lingüísticos» (con lo que toda su teoría se derrumbaria) o son sólo proyecciones intra-paradigmáticas (con lo que también toda su teoría se deriumbaría). En definitiva, la apelación de Fish a la "retórica" deja aún pendiente el problema de cómo identificar y distinguir tales «comunidades interpretativas»; cs decir, no alcanza aún a explicar los mecanismos de cómo las mismas emergen, se modifican y transforman históricamente.

Esto parece devolvernos a una problemática análoga a la planteada originariamente por Hirsch, es decir, la de la nece sidad de buscar tras los textos la huella del autor a fin de fijar su contenido semántico. Sin embargo, la quiebra de la hermenéutica se expresaría en una serie de desplazamientos decisivos. El más fundamental es que el "autor» al que se refiere Fish ya no es el de los textos analizados sino el de sus propios intérpretes. La legitimidad de la empresa crítica ya no se fundaría tanto en la posibilidad de acceder al significado <oculto» tras los textos, sino en su capacidad para dar cuenta de sus propios fundamentos epistémico-institucionales. Esto significa un desplazamiento del énfasis de los estudios desde los abjetos textuales y, eventualmentc, su relación con sus contextos particulares de emergencia, al de los mecanismos constructivos de tales objetos por parte de la crítica y de sus propias condiciones histórico-institucionales de producción. Llegado a este punto, la crítica se convierte en una metacrítica. Pero, para llegar a este grado de autorreflexividad, la misma debió pasar previamente por una serie de estadios sucesivos.

En la trayectoria descrita que lleva de Hirsch a Hoy vimos cómo la problematización de la relación entre el texto y su contexto de emergencia llevó primero a tematizar las relaciones entre aquéllos y su contexto de recepción. En un estadio subsiguiente, Fish viene finalmente a problematizar el propio contexto de recepción: este mismo se convierte entonces en objeto de interpretación por parte de las diversas y particulares comunidades interpretativas. Es entonces que emerge la pregunta relativa a las condiciones de producción e intercambio de la propia empresa crítica. Ésta debe, pues, volverse sobre sí, tornarse doblemente reflexiva, y comenzar a interrogarse respecto de los marcos tcórico-institucionales en los que la disciplina puede desenvolverse como tal; en fin, hacer explicitos sus fundamentos enraizados en los modos particulares en que los dicursos pueden hoy interpretarse y circular en el medio académico y en el tipo de exigencias que tal medio impone a sus miembros.

Sin embargo, según lo visto aquí, llegado a cste punto, la crítica parece enfrentarse a una serie de aporías que (como las observadas en el caso Fish) la tornan inevitablemente autocontradictoria. Ésta no parece capaz de dar cuenta de sus propios fundamentos. La quiebra de la hermeneutica parece, pues, arrastrar consigo la de la crítica literaria toda en una pendiente fatal hacia el Mäelstrom del relativismo más absoluto. Claro que todavía puede, quizás, argumentarse que tal conclusión relativista no es inescapable; que las aporias señaladas estarían simplemente revelando una limitación sólo inherente al presente ugiro lingüísticon: su incapacidad de servirse de sus propias categorias de análisis a fin de dar cuenta de su propia discursividad, es decir, de convertirse en su propio metadiscurso (tarea cuya realización nos exigiría movernos más allá del horizonte definido por la «lingüisticalidad»). De hecho, el relativismo no es tampoco sostenible, puesto que conduce siempre a la conocida «falacia relativista». De ser así, el presente «giro lingüístico» habría 
que considerarlo como un mero estadio hacia algún otro modo de la crítica susceptible de dar cuenta de la serie de problemas que ćste plantea sin llcgar a resolver. El presente «giro lingüístico» sería, pues, un ejemplo, quizás excepcional, de una corriente crítica que llega a definir una problemática a la que, sin embargo, las catcgorías de análisis que ella misma aporta no ofrecen respuestas válidas. En cualquiera de los casos, Fish parece estar en lo cierto (por perturbadora que resulte esta conclusión, a la que él mismo resistió aunque sin éxito) cuando afirma que éste ha hecho ya imposible un simple regreso al tipo de certidumbres que acompañaron la época de florecimiento de la hermenéutica, certidumbres a las que (como la idea de la transparencia al autor de la significación de su obra) las nuevas corrientes críticas vinieron definitivamente a desmantelar desde el momento en que pusieron de manifiesto aquello que hasta entonces aparecía como sus aprions (y, por lo tanto, impensables dentro de su horizonte de pensamiento dado): los mecanismos constructivos de la propia crítica y su relación con aquellos marcos epistémico-institucionales dentro de los cuales la actividad crítica puede desenvolverse en tanto que disciplina académica.

\section{NOTAS}

1 La New Critical School forece en los años 1930 y 1940 (aunque reconoce sus antecedentes en los escri tos de la década anterior de T.S. Eliot, I. A. Richards y Wiltiam Empsan, en Inglaterra, y Crowe Ransom y Allen Tate en Esiados Unidos). Además de los mencionados, participaron de la misma R. P. Blackmur, Cleanth Brooks, René Wellek, W. K. Winters y, en alguna medida también, Kenneth Burke. F. R. Leavis, y Yvor Winters. El movimiento contó con varias publicaciones afines, como The Cnterion (1922-29), dirigida por Eliot; Scrutiny (1932-53), dirigida por Leavis, en Inglaterra; la Southem Review (1935-42), editada por Brooks y Warren; la Canon Review (1938-59), dirigida por Ransam, y la Sewanee Review (1944-45), dirigida por Tate en Estados Unidos. Hacia la década siguiente, la New Critical School pierde su aura "revolucionaria" y pasa a integrarse al establishment. La escucla adopta un tono uprofesionalista", que se conecta con su enfoque formalista. Entonces se producen también sus textos teóricos más importantes, como Theory of Literature (1949), de Warten; The Verbal Icon (1954), de W. K. Wimsalt; The New Apologists for Poely (1956), de Krieger, y Literay Criticism: A Short History (1957), de Brooks y Wimsatt. Para una buena sintesis de las ideas fundamentales del grupo, ver la entrada «New Criticism m, escrita por Cleanth Brooks, en Alex Preminger et al. (ed.), Princeton Encyclopaedia of Poetry and Poetics (Princeton, Princoton University Press, 1974), pp. $567-568$.
- Sontag, Against Interpretation and Other Essays (Nueva York, Delta Books, 1964), p. 14. Sontag flue acompañada en su radicalismo contra el New Criticism y las escuelas tradicionales por autores diversos, como J. Hillis Miller, Paul Brodtkrob y Geoffrey Hartman.

1 Eric Donald Hirsch, Jr, (1928), es Whliam R. Kenan Professor of English en la Universidad de Virginia y miembro del Comité Editorial de New Literary Histony. Es también autor de inocence und Experience; An Introduction to Blake (New Haven, Yale University Press, 1964); Volidity in Interpretation (New Haven, Yale University Press, 1967); The Aims of Inteppetationt (Chicago, University of Chicago Press, 1976); The Phitosophy of Compasition (Chicago, University of Chicago Press, 1977); aThe Politics of Interpretations, en W. J. T. Mitchell (comp.), The Politics of Interpretation (Chicago, The University of Chicago Press, 1983), Pp. 321-334; «Beyond Convention?", New Literary Histony 14.2 (invierno 1983), pp. 389-398; «On Theories and Metaphors: A Comment on Mary Hesse's Papers, New Literary History, 17.1 (otoño 1985), pp. 49-59 (en ese mismo nútuero aparece la respuesta de Mary Hesse, pp. 57-60); "Transhistorical Intentions and the Persistence of Allegory", New Literary History, 25,3 (verano 1994), pp. 549-67. Ilirsch estudia actualmente asuntos relacionados con la enseñanza de la literatura.

- Hirsch es asociado con la «vicja hermenéuticas (Schleiermacher, Dilthey). La knueva hermenéutica» de Heidegger y Gadaner es introducida por Richard 
Palmer, Hermeneutics: Interpretation Theory in Schletermacher, Dilhey, Heidegger, and Gadamer (Eyanston, Northwestern University Press, 1969).

- David Couzens Hoy, The Critical Circle (Berkeley y Los Angeles, University of California Press, 1978). Hoy es profesor de Filosofia en la Universidad de California en Santa Cruz. Ha publicado: con Thomas McCarthy, Critical Theory (Oxford, Blackwcl, 1994); «ls Hermeneutics Ethocentric?n, en D. Hiley, J. Bohmand y R. Schusterman (comp.). The Interpretative Turr, Philosophy, Science, Cullure (Ithaca, Comell University Press, 1991), pp. 155-178, y aDeconstructing "ldeology"", Philosophy and Literature, 18.1 (abril 1994), pp. 117. Tambiên ha editado The Foucault Reader (Oxford, Blackwell, 1986).

s Ver W. K. Wimsatt, Ir., The Verbal Icon: Sudies on the Meaning of Poetry (Nueva York, Noonday Press. 1954). En particular el capitulo de Wimsait Jr. y M. Beardsley, kThe Intentional Fallacy».

${ }^{7}$ Stanley Eugene Fish, Is there a Text in this Class? The Authority of Interpretative Communities (Cambridge, Harvard University Press, 1980). Fish es Jefe del Departamento de Artes y Ciencias Inglesas, Profesor Distinguido de Literatura Inglesa y Profesor de Leyes en la Universidad de Duke. Otras obras de este autor son: John Sketton's Poetry (New Haven, Yale University Press, 1965); Surprised by Sin: The Reader in Paradise Lost (Berkelcy, University of California Press, 1971); Self-Consuming Artifacts; The Experience of Seventeenth-Century Literature (Berkeley, University of California Press, 1972); The Living Temple: Geonge Herbert and Catechizing (Berkeley, University of California Press, 1978); Doing What Comes Naturally: Change, Rhetoric, and the Practice of Theory in Literary and Legal Studies (Durham, Duke University Press, 1989), y There's no Such Thing as Free Speech, and It's $A$ Good Thirg, Too (Nucva York, Oxford University Press, 1994). Agunos de los estudios sobre la obra de Fish son: D. Patterson, aYou Made Me Do it. My Reply to Fish", Texas Law Review, 72.1 (noviembre 1993), pp. 67-77; R. Lanha, eIntroduction to Stanley Fish», Mition Quurterly, 26.3 (octubre 1992), pp. 9698; J. Foreman, «Theory, Language-Games, and Fish», New Orleans Review, 18.4 (invierno 1991), pp. 15-27; G. Currie «Text without Context, Some Errors of Stanley Fishs, Philosophy and Literature, 15.2 (octubre 1991), pp. 212-228; S. Barber, stanley Fish and the Future of Pragmatism in Legal Theory», University of Chicago Legal Review, 58.3 (verano 1992), pp. 1033-1043; J. Worthen, aOn the Matter of the Text (A Study on Stanley Fish's Literary Theory)", University of Toronto Quarterly, 60.3 (primavera 1991), pp. 337-353; C.Longines, «Power, Meaning and Per- suasion in Freud's the "Wolf-Man". A Response to Stanley Fishw, Minnesora Review, 34.3 (primavera 1990), pp. 118-134; M. Spikes, «A Kripkean Critique of Stanley Fish", Soundings, 73.2-3 (verano-otono 1990), pp. 327-341, y J. Michael, whetorical Ouestions in Stanley Fish's Daing what Comes Naturaly", Diacritics, 20.1 (verano 1990), pp. 54-73; R. Dworkín uMy Reply to Stanley Fish (and Walter Benn Michaels): Please Don't Talk About Objectivity Any Mores, en W. J. T. Mitchell (comp.), The Politics of Intepprtation (Chicago, The Chicago University Press, 1983). pp. 287-314.

" «o hay un modo único de lectura", dice Fish, sque sea correcto o natural, sólo modos de leer que son extensiones de las perspectivas de las respectivas comunidades» (is There a Text in this Class?, p. 16).

" Lo contrario supone, como señala Jay respecto de la noción de afusión de horizontes», la posibilidad de algo parecido a, según postulara Mannheim, «una totalización "relacionista" de puntos de vista en conflicto por parte de una intelligentsia colocada por cncima de los mismos (a free-floating intelligentsia) * (Jay, "Should Intellectual History?", en LaCapra-Kaplan, Modem European, p. 103),

Al respecto, ver la reseña del debate entre Habermas y Gadamer en Jay, whould Intellectual History?" (en LaCapra-Kaplan, Modern Furopear, pp. 89-110).

" Fish relata, en su introducción a is there a Text in this Class?. la historia de cómo fue también victima, en sus escritos tempranos, de una ambigüedad similar. "Lo que no veía es que no podía sostener consistentemente los dos argumentos al mismo tiempo... Cuando alguien me acusaba de que mi ćnfasis en el lector llevaba directamente al solipsismo y la anarquía, respondía insistiendo en las limitaciones impuestas sobre los lectores por el texto; y si alguien caracterizaba mi posición cono sólo la más reciente vuelta de la tuerca del New Crificism, replicuba que en mi modelo el lector era liberado de la tirania del texto y se le otorgaba un rol central en la producción de sentido. En síntesis, me estaba moviendo en dos direcciones (incompatibles) al mismo tiempo\% (pp. 7-8).

12 Stanley Fish, *Commentary: The Young and the Restlesss, en H. Aram Veeser, The New Historicism (Nueva York, Roulledge, 1989), p. 312.

1." Fish, "Anti-Protesionalism", New Lituray His" tony, 27.1 (atoño 1985), p. 91. Ver tambićn, en el mismo número (pp. 109-117), la respuesta de Gerald Graff (aInterpretation on Tlon: A Response to Stanley Fishm), seguida por la réplica de Fish (aA Reply to Gerald Graff», pp. 119-127). 